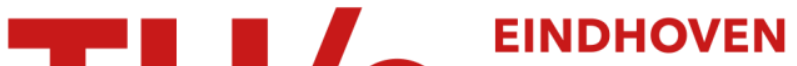 UNIVERSITY OF TECHNOLOGY
}

\section{Active tuning of the $\mathrm{g}$-tensor in InGaAs/GaAs quantum dots via strain}

Citation for published version (APA):

Tholen, H. M. G. A., Wildmann, J. S., Rastelli, A., Trotta, R., Pryor, C. E., Zallo, E., ... Silov, A. Y. (2019). Active tuning of the $g$-tensor in InGaAs/GaAs quantum dots via strain. Physical Review B, 99(19), [195305]. DOI:

10.1103/PhysRevB.99.195305

DOI:

10.1103/PhysRevB.99.195305

Document status and date:

Published: $16 / 05 / 2019$

Document Version:

Publisher's PDF, also known as Version of Record (includes final page, issue and volume numbers)

Please check the document version of this publication:

- A submitted manuscript is the version of the article upon submission and before peer-review. There can be important differences between the submitted version and the official published version of record. People interested in the research are advised to contact the author for the final version of the publication, or visit the $\mathrm{DOI}$ to the publisher's website.

- The final author version and the galley proof are versions of the publication after peer review.

- The final published version features the final layout of the paper including the volume, issue and page numbers.

Link to publication

\section{General rights}

Copyright and moral rights for the publications made accessible in the public portal are retained by the authors and/or other copyright owners and it is a condition of accessing publications that users recognise and abide by the legal requirements associated with these rights.

- Users may download and print one copy of any publication from the public portal for the purpose of private study or research.

- You may not further distribute the material or use it for any profit-making activity or commercial gain

- You may freely distribute the URL identifying the publication in the public portal.

If the publication is distributed under the terms of Article 25fa of the Dutch Copyright Act, indicated by the "Taverne" license above, please follow below link for the End User Agreement:

www.tue.nl/taverne

Take down policy

If you believe that this document breaches copyright please contact us at:

openaccess@tue.nl

providing details and we will investigate your claim. 


\title{
Active tuning of the $g$-tensor in InGaAs/GaAs quantum dots via strain
}

\author{
H. M. G. A. Tholen, ${ }^{1,}{ }^{*}$ J. S. Wildmann, ${ }^{2}$ A. Rastelli, ${ }^{2}$ R. Trotta,,${ }^{2,3}$ C. E. Pryor, ${ }^{4}$ E. Zallo, ${ }^{5,6}$ \\ O. G. Schmidt, ${ }^{5}$ P. M. Koenraad, ${ }^{1}$ and A. Yu. Silov ${ }^{1}$ \\ ${ }^{1}$ Department of Applied Physics and Institute for Photonic Integration, Eindhoven University of Technology, \\ P.O. Box 513, NL-5600MB Eindhoven, The Netherlands \\ ${ }^{2}$ Institute of Semiconductor and Solid State Physics, Johannes Kepler University Linz, Altenbergerstraße 69, A-4040 Linz, Austria \\ ${ }^{3}$ Department of Physics, Sapienza University of Rome, Piazzale A. Moro 5, 00185 Rome, Italy \\ ${ }^{4}$ Department of Physics and Astronomy and Optical Science and Technology Center, University of Iowa, Iowa City, Iowa 52242, USA \\ ${ }^{5}$ Institute for Integrative Nanosciences, IFW Dresden, Helmholtzstraße 20, D-01609 Dresden, Germany \\ ${ }^{6}$ Paul-Drude-Institut für Festkörperelektronik, Hausvogteiplatz, 5-7, 10117 Berlin, Germany
}

(Received 25 February 2019; revised manuscript received 26 April 2019; published 16 May 2019)

\begin{abstract}
Dynamic control over the full $g$-tensor in individual InGaAs/GaAs self-assembled quantum dots is achieved by inducing external strain via a piezoelectric actuator. The full $g$-tensor is obtained by measuring in different geometries with different angles between an externally applied magnetic field and the quantum dot growth axes. A large decrease in the out-of-plane hole $g$-factor with strain is observed, whereas the other components are found to be less sensitive. To further investigate this, a numerical model based on eight-band k.p-theory is used and an excellent agreement with the experimental results is established, both qualitatively and quantitatively. Furthermore, the calculations reveal the origin of the observed large change in the out-of-plane hole $g$-factor to be the increase in heavy-hole light-hole splitting under compressive stress.
\end{abstract}

DOI: 10.1103/PhysRevB.99.195305

\section{INTRODUCTION}

Quantum dots (QDs) [1] have often been suggested as fundamental building blocks for future quantum technology, as they can be exploited as sources of single photons or hosts of quantum bits [2]. The single electrons and holes confined to the QDs carry besides a charge also a spin, which can be used to store and process information [3]. Coupling this spin to an externally applied magnetic field leads to an energy difference between the two spin states called the Zeeman splitting. This splitting is generally linear for magnetic fields up to at least $10 \mathrm{~T}$, the highest investigated value in this work. The Zeeman splitting is characterized by the $g$-tensor, which is isotropic in free space. For the electron, the $g$-tensor then reduces to the well-known electron $g$-factor which has a value of roughly 2 . However, in the case of a semiconductor, the envelope part of the wave function causes substantial deviations from the free electron $g$-factor of 2 , all the way down to -51.3 for $\mathrm{InSb}$ [4]. Bulk electron $g$-factors for the materials used in this research, InAs and GaAs, are -14.7 and -0.44 , respectively $[5,6]$. In the lens-shaped QDs investigated in this work, the asymmetric shape leads to an anisotropic $g$-tensor. Gaining control over this $g$-tensor is valuable as it implies gaining control of the Zeeman splitting and therewith the spin states.

The spin of a charge carrier can be fully controlled if one of the components of the $g$-tensor can be tuned around zero [7]. It is well-known that the components of the $g$-tensor depend strongly on the exact electronic structure of the investigated system. The size- and shape-dependency of the $g$-tensor has

\footnotetext{
*h.m.g.a.tholen@tue.nl
}

been thoroughly investigated in the past for different QD systems [8-12], however these parameters cannot be dynamically controlled post-growth. Therefore, a method to in-situ change the electronic structure of the QD is required, for instance by means of an externally applied electrical or elastic stress field.

Efforts have been made to achieve $g$-tensor manipulation using external electric fields in InGaAs QDs [13-19], however to our best knowledge, an experimental demonstration of tuning any of the components around zero has not yet been achieved. We therefore investigate the strain-dependence of the $g$-tensor. The influence of strain on the electronic structure of a single QD is quite well-known and has been thoroughly investigated in the past, both experimentally and theoretically [20-25]. In previous work we have already shown a strong dependence of the out-of-plane exciton $g$-factor on strain [26]. In the current work we resolve the full $g$-tensor for both the electron and hole separately.

\section{THEORETICAL BACKGROUND}

Assuming a cylindrical symmetry of our structure the $g$ tensor reduces to

$$
\boldsymbol{g}_{e, h}=\left(\begin{array}{ccc}
g_{e, h}^{\|} & 0 & 0 \\
0 & g_{e, h}^{\|} & 0 \\
0 & 0 & g_{e, h}^{\perp}
\end{array}\right),
$$

where $g_{e, h}^{\|}$and $g_{e, h}^{\perp}$ are the in-plane and out-of-plane electron and hole $g$-factors, respectively. Each individual component has two different contributions according to

$$
g_{\text {tot }}=g_{s}+g_{\text {orb }}
$$


where $g_{\text {tot }}$ is the total $g$-factor and $g_{s}$ and $g_{\text {orb }}$ denote the spin part and the orbital part of the $g$-factor. The latter plays a significant role due to spin-orbit coupling, which creates spin-correlated currents [27]. These currents create a spindependent magnetic moment which couples to the magnetic field, leading to the orbital contribution to the $g$-factor. Moreover, the confinement in a QD leads to a quenching of this orbital momentum, decreasing its contribution. This concept will prove to be important in interpreting the results presented in this work.

To show how an external magnetic field affects the system, we have to consider the Zeeman Hamiltonian, which governs the splitting of states with magnetic field and is given by

$$
H_{\text {Zeeman }}=\frac{1}{2} \mu_{B} \boldsymbol{B} \cdot \boldsymbol{g} \cdot \boldsymbol{\sigma} .
$$

Here $\sigma$ is a vector $\left(\sigma_{x}, \sigma_{y}, \sigma_{z}\right)$ containing the Pauli matrices, $\boldsymbol{g}$ is the $g$-tensor from Eq. (1), and $\boldsymbol{B}$ is an externally applied magnetic field. For a rotationally invariant QD, an arbitrary magnetic field can always be written as $\boldsymbol{B}=$ $(\sin \beta, 0, \cos \beta) B_{0}$, where $B_{0}$ is the amplitude of the field and $\beta$ the angle between the field and the excitation/collection direction. The Zeeman Hamiltonian and the corresponding energies then become

$$
\begin{aligned}
& H_{\text {Zeeman }}=\frac{1}{2} \mu_{B}\left(\begin{array}{cc}
g^{\perp} \cos \beta & g^{\|} \sin \beta \\
g^{\|} \sin \beta & -g^{\perp} \cos \beta
\end{array}\right) B_{0}, \\
& E_{\text {Zeeman }}= \pm \frac{1}{2} \mu_{B} \sqrt{\left(g^{\|} \sin \beta\right)^{2}+\left(g^{\perp} \cos \beta\right)^{2}} B_{0} .
\end{aligned}
$$

Since we are interested in the $g$-factors both out-of-plane and in-plane, two limiting cases are to be discussed. Applying the magnetic field parallel to the growth direction of the QD ( $\beta=0^{\circ}$, Faraday geometry) will yield the out-of-plane $g$-factors, whereas applying the magnetic field perpendicular to the growth direction of the $\mathrm{QD}\left(\beta=90^{\circ}\right.$, Voigt geometry) will yield the in-plane $g$-factors.

When applying an external magnetic field, a charge carrier is not only affected by the Zeeman effect. On top of this, there is a diamagnetic contribution to the energy, quadratic in magnetic field and independent of spin. This originates from a magnetic-field-induced magnetic moment which couples back to the applied magnetic field. This contribution will show up in the measurements, however, it will not be discussed any further.

As both the electron and hole energy levels split up simultaneously, in principle four transitions are possible, as indicated in Fig. 1(c). However, in the Faraday geometry, angular momentum conservation has to be considered. As a photon carries away an angular momentum of one, only the two transitions with a total combined spin of \pm 1 are optically active (bright states), whereas the other two are not optically active (dark states). The two visible transitions will emit right-handed circularly polarized $\left(\sigma^{+}\right)$and left-handed circularly polarized $\left(\sigma^{-}\right)$photons for the +1 and -1 transitions, respectively. As a result, the PL spectrum will show two peaks at non-zero magnetic field and the splitting $\Delta E_{\text {Zeeman }}$ between the two is given by

$$
\Delta E_{\text {Zeeman }}=E\left(\sigma^{+}\right)-E\left(\sigma^{-}\right)=\mu_{B} B_{0} g_{\mathrm{exc}}^{\perp},
$$
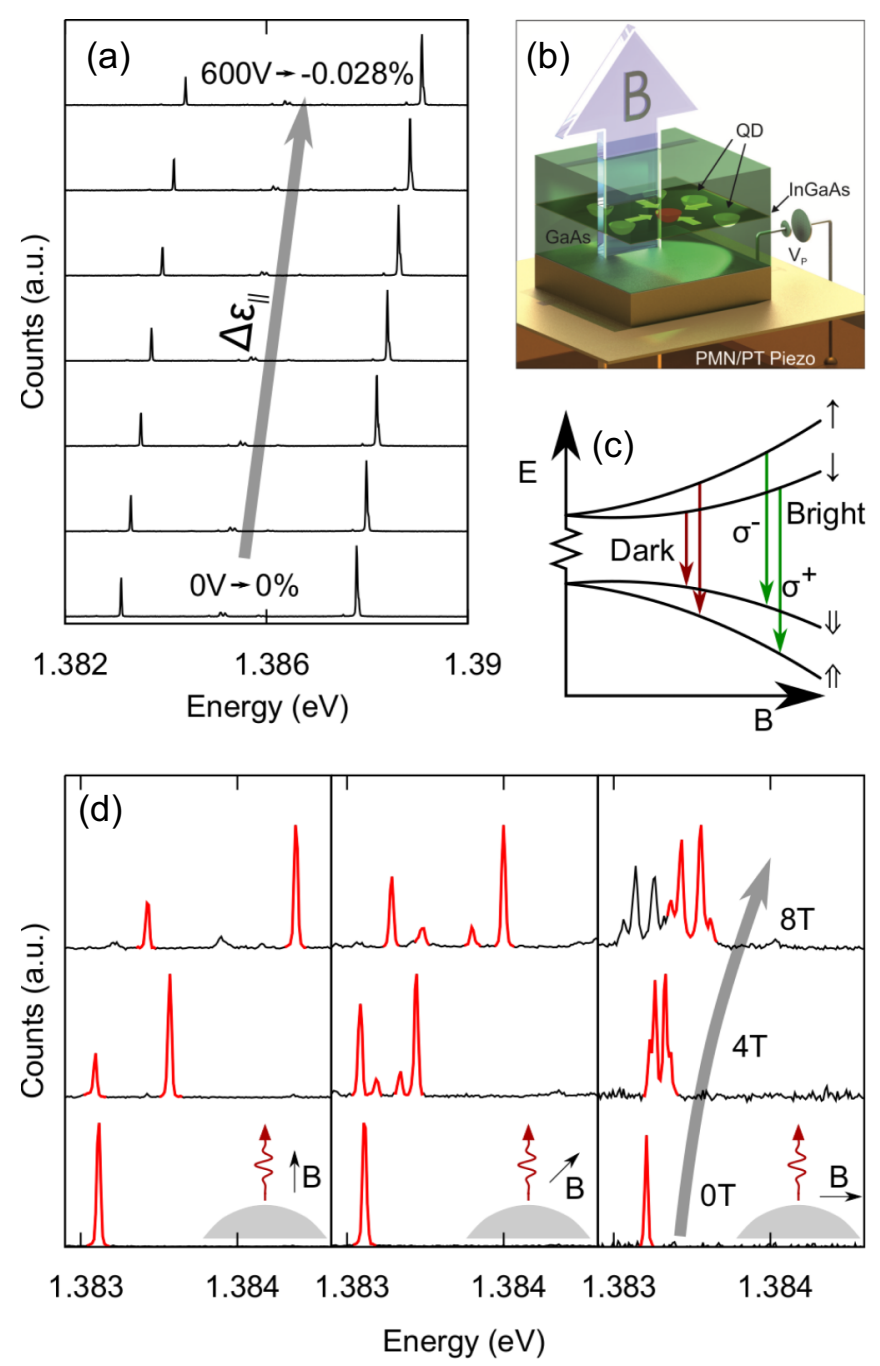

FIG. 1. (a) Spectrum of a single QD as a function of the strain induced by a piezoelectric actuator. Each subsequent spectrum represents an increase in the actuator voltage of $100 \mathrm{~V}$, corresponding to an increase in compressive strain of roughly $0.045 \%$. The spectral lines experience a blue-shift of roughly $1.3 \mathrm{meV}$ as the voltage (and hence magnitude of compressive strain) is increased. Strain values are an estimate based on theory. (b) Schematic overview of the sample. (c) Schematic overview of the splitting of the energy levels as a function of the applied magnetic field. Both the electron and hole experience a Zeeman splitting linear in $\mathrm{B}$ and a diamagnetic shift quadratic in B. (d) Excitonic emission as a function of magnetic field, with the field applied in the growth direction (left), at an angle of 45 degrees (middle) and in-plane (right), as shown in the insets. The expected behavior is reproduced.

where $g_{\mathrm{exc}}^{\perp}=g_{h}^{\perp}+g_{e}^{\perp}$ is the exciton $g$-factor. The sign of the exciton $g$-factor can then be determined by measuring the polarization of the separate peaks.

In the Voigt geometry, the symmetry of the QD is broken and the bright and dark states mix, revealing four linearly polarized transitions [28]. This yields two Zeeman splittings given by

$$
\Delta E_{\text {Zeeman }}= \pm \mu_{B} B_{0}\left(g_{h}^{\|} \pm g_{e}^{\|}\right)
$$


As it is impossible in our experiment to assign the peaks to a specific linear polarization (see Sec. III A), this only yields absolute values for the electron and hole $g$-factor. To be able to separate the electron and hole $g$-factor in the Faraday geometry as well, an additional measurement at an intermediate angle $\beta$ has to be performed. However, as the $g$-factor only appears squared in Eq. (5) this does not resolve the issue of sign assignment. This will be addressed later. For the intermediate measurement, we choose $\beta=45^{\circ}$.

In this work external strain is induced by means of a piezoelectric actuator. The InGaAs QDs are grown by molecular beam epitaxy and contained in a thin membrane with a thickness of about $300 \mathrm{~nm}$. Due to the procedure used during capping [29], the QDs have a maximum height of about $2.5 \mathrm{~nm}$ and a radius between 10 and $15 \mathrm{~nm}$. The In-concentration is estimated to be between 20 and $40 \%$. The membranes are transferred to the piezoelectric actuator via flip-chip and gold thermocompression bonding techniques. The used piezoelectric is $\left[\mathrm{Pb}\left(\mathrm{Mg}_{1 / 3} \mathrm{Nb}_{2 / 3}\right) \mathrm{O}_{3}\right]_{0.72}-$ $\left[\mathrm{PbTiO}_{3}\right]_{0.28}$ (PMN-PT). The detailed growth and fabrication processes are described elsewhere [30]. The resulting sample structure is schematically shown in Fig. 1(b).

The piezoelectric actuator is poled in such a way that applying a positive voltage $V_{p}$ across the piezo induces a biaxial compressive strain. This strain is then transferred to the membrane containing the QDs. The amount of applied strain depends strongly on the quality of the bond between the piezo and the nanomembrane. Therefore, we achieve a maximum strain of $\Delta \epsilon_{\|}=-0.03 \%$, whereas values up to $\pm 0.2 \%$ have been reported elsewhere [30]. The value of strain is determined by comparing the shift in emission energy with other work $[20,30]$ and our numerical calculations, which will be discussed in the second part of this paper.

Figure 1(a) shows the PL spectrum of a single QD as a function of the applied strain. The two different spectral lines originate from different excitonic complexes. The lack of fine structure splitting (within our spectral resolution of $<30 \mu \mathrm{eV}$ ) in any of the lines at $B=0 \mathrm{~T}$ validates our assumption for the cylindrical symmetry of our QDs in Sec. II [28]. Both lines exhibit a linear blue-shift of around $1.3 \mathrm{meV}$. As previous research has shown no significant difference in strain-dependence between the different excitonic complexes $[20,26]$, assignment of the complexes is irrelevant and it suffices to analyze only one of the lines.

\section{EXPERIMENTAL RESULTS}

To experimentally determine the full $g$-tensor for both the electron and hole of single QDs, a magneto-optic setup is used. The setup is equipped with a superconducting magnet, allowing for magnetic fields up to $10 \mathrm{~T}$. The sample is cooled down to a temperature of $4 \mathrm{~K}$ and an objective is located directly above the sample for excitation and subsequent PLcollection. The QDs are excited with a 635-nm laser and the PL is analyzed with a spectrometer and a Silicon CCD. The different geometries are achieved by mounting the sample under different angles and rotating the objective accordingly.

Figure 1(d) shows one of the emission lines of a single QD for different geometries at different magnetic fields. The Faraday geometry reveals the expected doublet-splitting, where the other two geometries show a quadruplet-splitting, highlighted in red. It is not unusual to see additional complexes which are activated at higher magnetic fields, as is the case at $8 \mathrm{~T}$ in the Voigt geometry. These complexes however do not prevent us from tracking the spectral lines from $0 \mathrm{~T}$ all the way up to $10 \mathrm{~T}$. By simultaneously fitting Eq. (5) to the Zeeman energies extracted from the data presented in Fig. 1(d), the absolute values of the electron and hole $g$-factors can be determined. For this QD this yields $\left|g_{h}^{\perp}\right|=1.56 \pm 0.01,\left|g_{h}^{\|}\right|=$ $0.14 \pm 0.01,\left|g_{e}^{\perp}\right|=0.46 \pm 0.01$ and $\left|g_{e}^{\|}\right|=0.4 \pm 0.01$.

\section{A. Sign assignment}

Some extra analysis is required to determine the signs of the $g$-tensor components. The sign is defined in such a way that when a magnetic field is applied along a certain axis and the state with a negative total-momentum projection onto this axis is the ground state, the $g$-factor is considered to be positive [31].

For the out-of-plane $g$-factors this analysis is relatively straightforward. From the splitting of the two peaks the absolute value of the exciton $g$-factor can be determined. By determining the polarization of the peaks, the sign of the exciton $g$-factor can be directly determined according to Eq. (6). The signs of the electron and hole $g$-factors separately are then automatically also known. In the studied QD, both are negative, i.e., $g_{e}^{\perp}=-0.46$ and $g_{h}^{\perp}=-1.56$.

For the in-plane components the situation is more complicated. In the Voigt geometry, an external magnetic field leads to a linear polarization. The two different linear polarizations can be distinguished, but not unambiguously assigned to either of the peaks. The sign can therefore not be determined in this way. We therefore have to extract the signs from the numerical calculations, which will be presented in the second part of the paper. These reveal the sign of both the in-plane electron and hole $g$-factors to be negative.

\section{B. Strain-dependence}

Repeating the measurement described in Fig. 1(d) while varying the voltage applied to the piezoelectric actuator, the strain-dependence of the $g$-factors can be obtained. The result is shown in Fig. 2.

The $g$-tensor for the hole shows a remarkable anisotropy; the out-of-plane hole $g$-factor exhibits a relatively large shift, whereas the in-plane value is virtually unaffected. As the amount of strain varies from membrane to membrane and the shift in emission energy scales linearly with the induced strain, the sensitivities of the $g$-tensor components (i.e., the slopes of the lines in Fig. 2) are given in terms of the shift in emission energy. This also allows for an easier comparison with the calculations later on. The slopes are $\delta g_{h}^{\perp} / \delta E=$ $0.051 \mathrm{meV}^{-1}$ and $\delta g_{h}^{\|} / \delta E=0.001 \mathrm{meV}^{-1}$. The opposite anisotropy occurs for the electron, however, less pronounced. The electron $g$-factor shows only a limited change in both directions, with slopes of $\delta g_{e}^{\perp} / \delta E=-0.001 \mathrm{meV}^{-1}$ and $\delta g_{e}^{\|} / \delta E=0.005 \mathrm{meV}^{-1}$. To investigate the consistency of this result, in total five different single QDs are studied. The emission energies, $g$-factors and their slopes of all investigated dots are listed in Table I. Due to the limited spread in the 


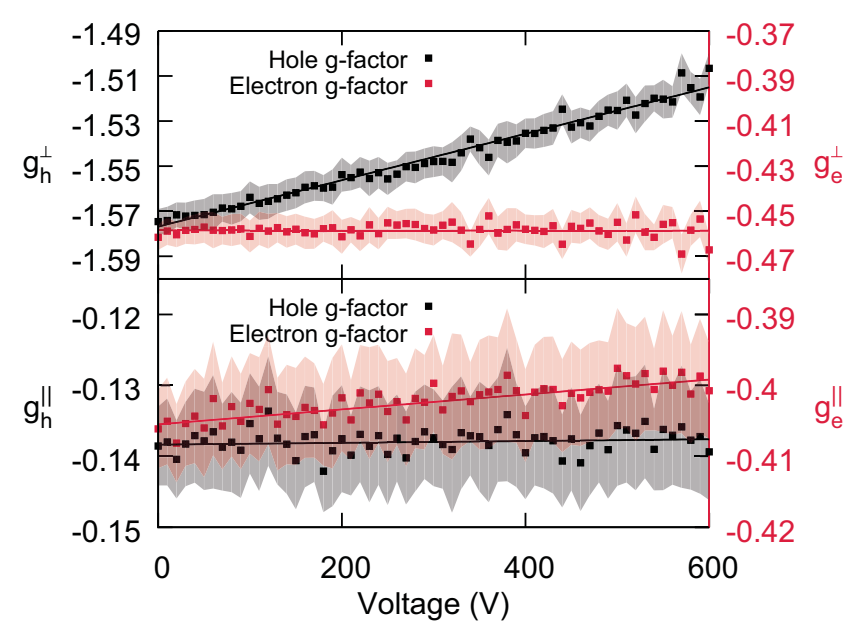

FIG. 2. Experimentally determined values for the electron (black) and hole (red) $g$-factors, both out-of-plane (upper panel) and in-plane (lower panel) as a function of voltage applied to the piezoelectric actuator. The values are obtained by combining results for the Zeeman splitting in three different geometries, applying magnetic fields up to $10 \mathrm{~T}$. Shaded areas indicate the error range.

absolute values of the $g$-factors, the sign assignment for the QD in Fig. 2 is also valid for the rest of the dots.

In the following, we will discuss each component of the $g$-tensor separately;

$g_{h}^{\perp}$ : The out-of-plane hole $g$-factor shows by far the largest sensitivity to the applied strain, with the slope ten times larger than any of the other components. The result is consistent for all dots, with slopes ranging roughly from $0.04-0.05 \mathrm{meV}^{-1}$. The observed shift is remarkably large considering the limited amount of strain delivered to the QD. The nominal values of $g_{h}^{\perp}$ range from -2.4 to -1.6 . This relatively wide spread is not unusual, as the hole $g$-factor is known to be extremely sensitive to the exact size, shape, composition and built-in strain of the QD.

$g_{h}^{\|}$: The in-plane hole $g$-factor shows much smaller nominal values, ranging from -0.15 to -0.3 . This anisotropy is actually well-known for a heavy hole, which is the dominating character in the ground state hole wave function due to the symmetry of the dot. The sensitivity to strain is therefore also smaller, however, the slopes show a rather large spread, even changing sign for one of the dots.

$g_{e}^{\perp}$ : For most dots, the out-of-plane electron $g$-factor shows no significant sensitivity to strain. The nominal value of $g_{e}^{\perp}$ shows only a small spread, with all values being around -0.45 .

$g_{e}^{\|}$: The in-plane electron $g$-factor shows the same sensitivity to strain in all dots, with a shift of $0.005 \mathrm{meV}^{-1}$. The nominal value is of the same order as $g_{e}^{\perp}$.

\section{CALCULATIONS}

To gain more insight in the physics behind the system, numerical calculations are performed. The calculations are based on an eight-band strain-dependent $\boldsymbol{k} \cdot \boldsymbol{p}$ model which is applied to a representative structure, a lens-shaped $\operatorname{In}_{0.4} \mathrm{Ga}_{0.6} \mathrm{As}$ QD with a height of $2.5 \mathrm{~nm}$ and a radius of $10 \mathrm{~nm}$. The initial strain due to lattice mismatch is calculated on a cubic grid using continuum elasticity theory. Additional isotropic biaxial strain is incorporated by introducing an additional layer under the grid and varying the lattice constant of this layer. It is confirmed that this approach induces the expected biaxial strain in the rest of the grid. The electronic calculations are then performed on the same grid as the strain calculations. Material parameters for InAs and GaAs were taken from Ref. [32]. Parameters for the alloy were determined by linear interpolation (Vegard's law), except when bowing parameters were available from Ref. [32]. Exchange and Coulomb interactions are neglected, as their contributions are small compared to the magnetic field contribution. The $g$-factors were determined by computing the Zeeman splitting in a uniform magnetic field. Further details of the model can be found in Refs. [33-36].

First we calculate the emission energy of the dot as a function of the additional biaxial compressive strain. The result is shown in the top panel of Fig. 3 and reveals a shift of $4.7 \mathrm{meV}$ at $-0.1 \%$ strain, which is consistent with former experimental results on similar dots $[20,30]$. This is thus used as a calibration [see also values quoted in Fig. 1(a)] for the achieved strain. The experimental shifts of 1.3-1.6 meV correspond with a strain of roughly $0.027-0.034 \%$. By including a magnetic field and tracking the Zeeman splitting, the $g$-tensors for the electron and hole can be independently calculated as a function of strain. The result is shown in Fig. 3.

\section{DISCUSSION}

The calculations nicely reproduce the strong sensitivity of the out-of-plane hole $g$-factor to applied strain compared to the other components. To quantitatively compare the results, Table II shows the values of the slopes $\left(\frac{\delta g}{\delta E}\right)$ for all

TABLE I. Emission energy, $g$-factors, and their sensitivities for five different dots, showing the consistency of the results. Sensitivities for the $g$-factors are given in change per eV of shift in emission energy.

\begin{tabular}{|c|c|c|c|c|c|c|c|c|c|}
\hline Dot & $\begin{array}{c}\text { Emission energy } \\
\mathrm{eV}\end{array}$ & $g_{h}^{\perp}$ & $g_{h}^{\|}$ & $g_{e}^{\perp}$ & $g_{e}^{\|}$ & $\delta g_{h}^{\perp} / \delta E$ & \multicolumn{2}{|c|}{$\mathrm{eV}^{-1}$} & $\delta g_{e}^{\|} / \delta E$ \\
\hline 1 & 1.383 & 1.560 & -0.137 & -0.460 & -0.402 & 51 & 1 & -1 & 5 \\
\hline 3 & 1.380 & 2.348 & -0.270 & -0.403 & -0.539 & 49 & 8 & 0 & 5 \\
\hline 4 & 1.385 & 1.705 & -0.207 & -0.432 & -0.405 & 41 & 7 & -1 & 5 \\
\hline 5 & 1.377 & 1.738 & -0.161 & -0.524 & -0.310 & 40 & -8 & 3 & 5 \\
\hline
\end{tabular}



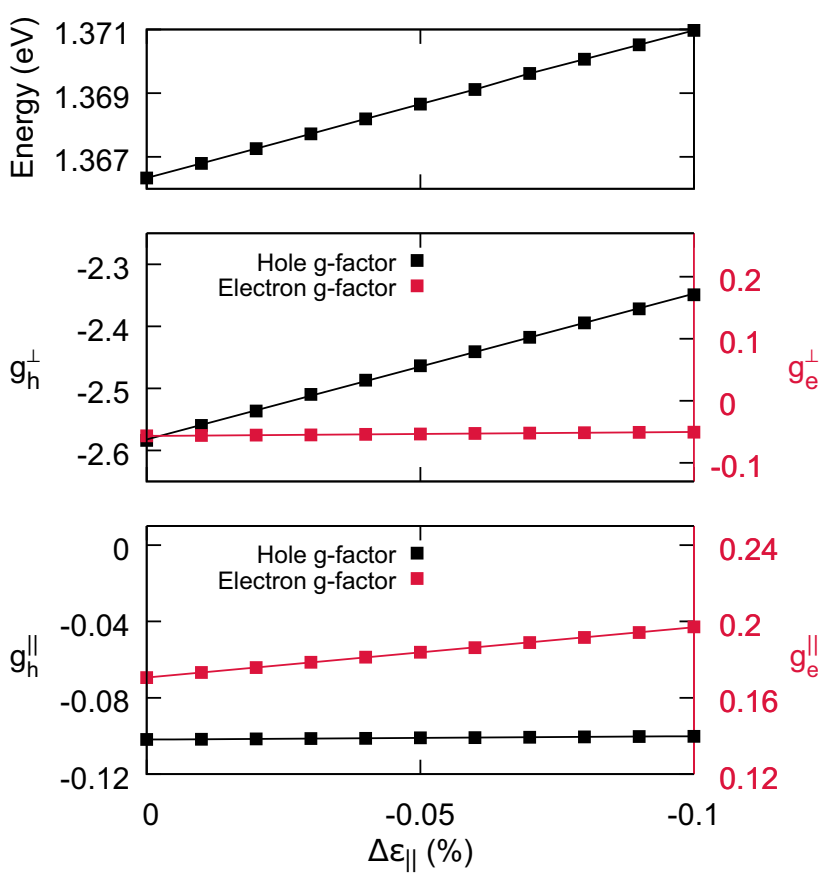

FIG. 3. Upper panel: Emission energy of the QD as a function of biaxial compressive strain. Middle and bottom panels: Calculated components of the electron and hole $g$-factors, out-of-plane and in-plane respectively. Values for the $g$-factors are computed by calculating the eigenenergies in a magnetic field of $10 \mathrm{~T}$.

components for both the calculations and the averaged experimental values.

We should note that there are some discrepancies in the absolute values of the $g$-factors. The geometries used are an estimate for the real dots, but are probably not $100 \%$ accurate. By exploring the parameter space around this typical dot, the $g$-factor components can be tuned closer to the experimental values. However, when varying the dot height from 1.5 to $5 \mathrm{~nm}$, the dot radius from 5 to $15 \mathrm{~nm}$ and the Indium concentration from 30 to $50 \%$, no significant changes $(<10 \%)$ in the sensitivities of the different components were observed. Therefore we choose not to optimize the dot parameters, since the observed sensitivities are quite robust against a variation of them.

The sensitivities of all components agree very well with the previously obtained experimental values. The numerical model can therefore be employed to gain insight into the physical origin of the change in $g$-factors. Since every wave

TABLE II. Values of the $g$-factors and their sensitivities to strain determined from both the numerical calculations and the experiment. The sensitivities $\delta g / \delta E$ show a good agreement between the two.

\begin{tabular}{l|rc|cc}
\hline \hline Component & Value & $\begin{array}{c}\delta g / \delta E \\
\mathrm{eV}^{-1}\end{array}$ & Value & $\begin{array}{c}\delta g / \delta E \\
\mathrm{eV}^{-1}\end{array}$ \\
\hline$g_{h}^{\perp}$ & -2.584 & 51 & -1.842 & 43 \\
$g_{h}^{\|}$ & -0.102 & 0 & -0.218 & 2 \\
$g_{e}^{\perp}$ & -0.057 & 1 & -0.445 & 1 \\
$g_{e}^{\|\|}$ & 0.171 & 6 & -0.394 & 5 \\
\hline \hline
\end{tabular}

function is a superposition of the Bloch states of all bands, the exact value of the $g$-factors originates from an interplay between those bands. Therefore, the composition of the electron and hole ground states has to be considered. Furthermore, the confinement of the wave functions will quench the orbital momentums associated with the $g$-factors and has therefore to be taken into account. We will discuss the electron and hole separately.

\section{A. Electron}

For the electron, the anisotropy in the sensitivity of the $g$-tensor is very well reproduced, as is clear from Table II. The out-of-plane $g$-factor experiences only a slight increase with applied strain, changing only by 0.001 for every $\mathrm{meV}$ of shift in the emission energy, whereas the in-plane component shows a larger sensitivity, changing by 0.005 per meV.

To gain insight into the origin of the increase in electron $g$-factors and the anisotropy between the two components, we have to consider two separate contributions which cause the electron $g$-factor to deviate from the free electron value of 2 ; the mixing of the valence band into the electron ground state and the quenching of the orbital momentum.

\section{Valence band mixing}

Spin-orbit coupling causes a (negative) deviation from the spin $g$-factor of 2, according to

$$
g_{e}=2-g_{\text {orb }} .
$$

However, since the conduction band is made up of $s$ orbitals which do not have an angular momentum, it does not contribute to the orbital part of the $g$-factor. A pure conduction band state would therefore have $g_{\text {orb }}=0$ leading to a $g$-factor of 2. However, due to the coupling between the conduction band and the valence band, the valence bands mix into the electron ground state. The $p$-states of the valence band, which have a nonzero angular momentum, then lead to a nonzero $g_{\text {orb }}$.

When investigating the composition of the wave function, we find that the electron ground state wave function has roughly $96 \%$ conduction band character. The remainder is made up of the valence band. When applying $-0.1 \%$ strain, the $\mathrm{CB}$ character decreases by $0.03 \%$. This change is negligible and contradicts the observed behavior, especially for the in-plane component, as it would lead to an increase of the VB contribution and therewith a decrease in the electron $g$-factor. We can therefore rule this out as the main mechanism behind the observed strain-sensitivity.

\section{Orbital momentum quenching}

As mentioned earlier, the orbital contribution to the magnetic moment (and hence the $g$-factor) originates from the spin-orbit interaction which gives rise to spin-correlated currents. By lifting orbital degeneracies, the confining potential limits the coherent mixing of different orbital states and therefore decreases the contribution of the orbital current to the magnetic moment. This mechanism is referred to as orbital angular momentum quenching $[35,36]$. In the presence of an 
applied magnetic field the current contributing to the magnetic moment is perpendicular to the field, and therefore the $g$-factor will be affected by the confinement transverse to the applied field.

Asymmetry in the confining potential leads to anisotropy in the angular momentum quenching, and hence in the $g$-factor. Such anisotropy has been observed in InAs/InP QDs [27,37]. With a radius $r$ that is much larger than the height $h$, our dots deviate significantly from spherical symmetry, however, the $g$-factor is less anisotropic than in Refs. [27,37], indicating weaker confinement. The weak anisotropy seen in both our experiments and calculations indicates electrons in our dots are weakly confined to the QD.

The barrier height does not alone determine the confinement strength. For example, a nominally high barrier will provide only weak confinement if the well is so small that the energy of the confined state is close to the energy of the barrier material. For this reason, it is advantageous to evaluate the degree of confinement by examining the wave function and its penetration into the barrier material. The lateral extension of the electron wave function is affected by strain in two ways. Firstly, biaxial compressive strain will decrease the dot radius and through the Poisson effect increase the height of the dot. This effect is relatively small as the maximum strain used in the calculations is only $0.1 \%$ and can therefore be neglected. More importantly, strain shifts the edge of the conduction band, which will consequently affect the degree of confinement of the electron state in the dot. The shift in the bandedge with strain is given by $\Delta E_{c}=a_{c} \epsilon$, where $a_{c}$ is the material specific hydrostatic deformation potential and $\epsilon=\epsilon_{x x}+\epsilon_{y y}+\epsilon_{z z}$ is the trace of the strain tensor. Since we are dealing with biaxial compressive strain, $\epsilon<0$ in our system. The values of $a_{c}$ for GaAs and InAs are $-7.17 \mathrm{eV}$ and $-5.08 \mathrm{eV}$, respectively [32], resulting in a larger increase of the conduction bandedge for the GaAs barrier compared to the InGaAs dot, i.e., the barrier is raised and the electron is more strongly confined. This effect has been confirmed experimentally on similar dots, where it has been shown to give rise to the increase of the biexciton binding energy upon compression [21].

To further illustrate this, Fig. 4 shows the probability density $|\psi|^{2}$ for both the electron and hole on cross-sections of the dot. It is evident that the electron shows weaker confinement compared to the hole. Especially in the growth direction, the leakage into the GaAs barrier is significant. By integrating $\left|\psi_{e}\right|^{2}$ over the dot material, we find that roughly $50 \%$ of the probability density of the wave function is accommodated in the barrier rather than the dot. Figure 5 shows how this develops when applying strain. The part of the probability density that is confined inside the dot increases by roughly $1 \%$ when the induced strain is $-0.1 \%$. This is directly caused by an increase in the confinement energy, which is shown on the right axis and given by the energy difference between the electron ground state and the conduction bandedge of the surrounding barrier.

To conclude, due to an increase in electron confinement, the extension of the electron wave function into the surrounding GaAs barrier decrease, leading to a smaller orbital contribution and an increase in the electron $g$-factor. As the leaking of the wave function into the barrier is more pronounced in the

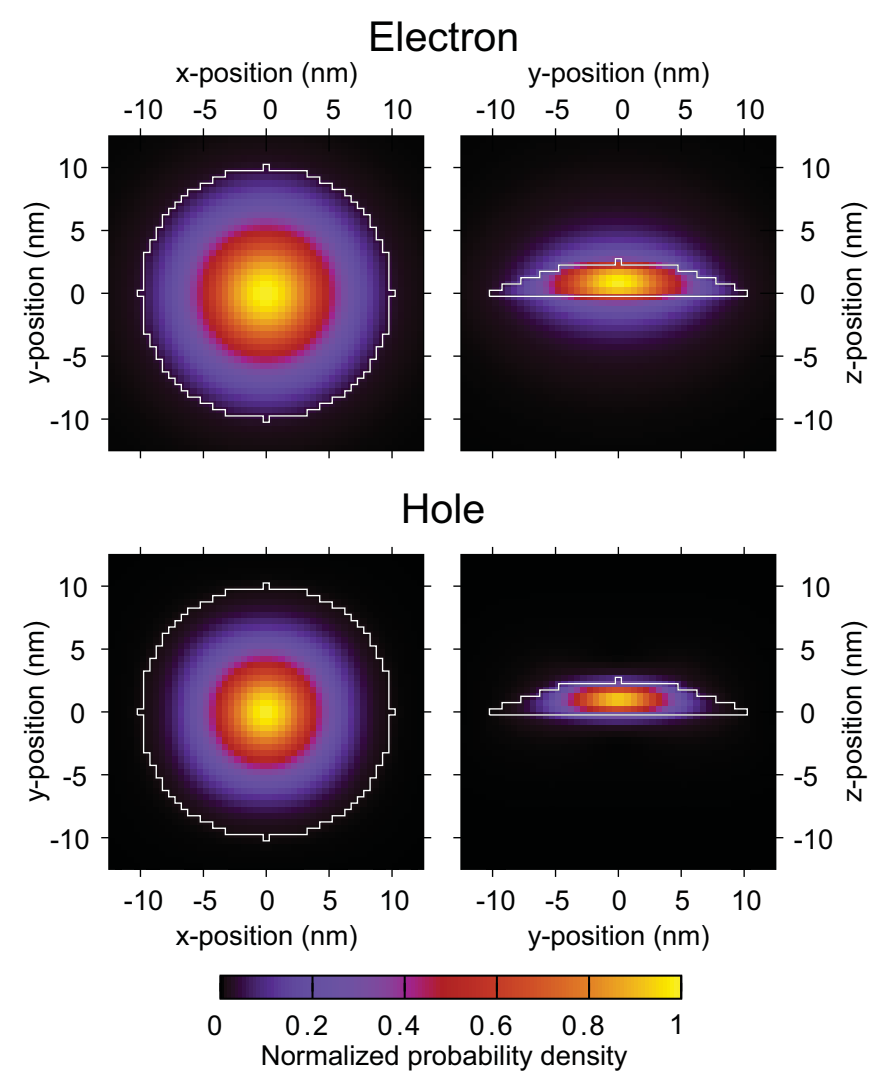

FIG. 4. Probability density $\left(|\psi|^{2}\right)$ for both the electron and hole wave functions on cross-sections of the dot. For clarity, each plot has been normalized to its maximum value. The left panel shows the footprint of the dot, the right panel shows the intersection plane perpendicular to the footprint and through the center of the dot.

growth direction, the in-plane electron $g$-factor will be most affected, which is indeed what we observe.

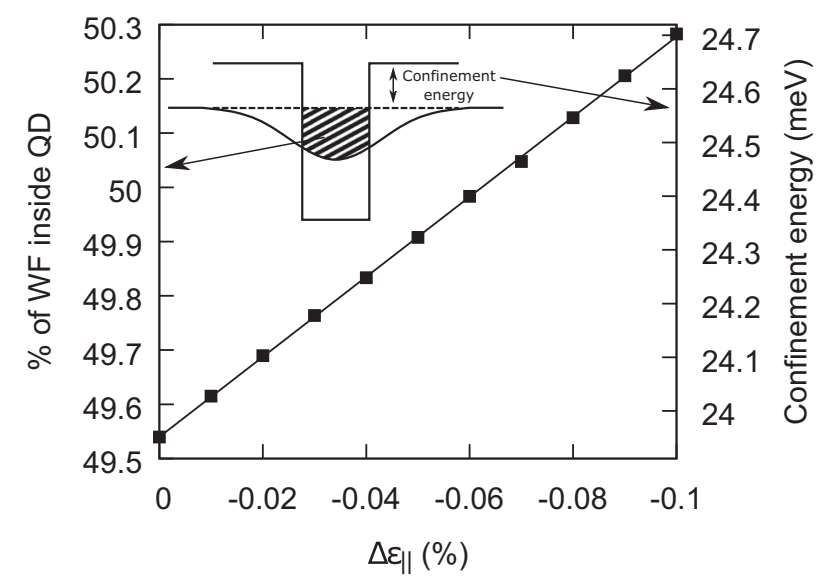

FIG. 5. $|\psi|^{2}$ integrated over the QD as a function of applied strain, indicated by the shaded region in the inset. The right axis shows the corresponding confinement energy, which is the difference between the electron ground state level and the conduction bandedge of the GaAs barrier, as shown in the inset. 


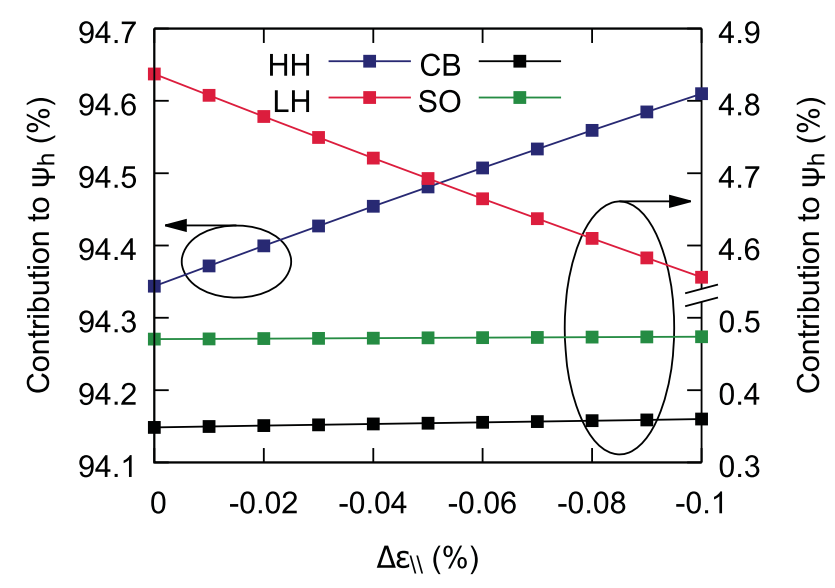

FIG. 6. Contribution of the different bands to the hole ground state wave function as a function of the applied strain. The contribution is determined by computing $\left|\psi_{n}, h\right|^{2} \mid$ where $n$ denotes the band. Note that the HH-contribution is associated to the left axis, while the other contributions are associated to the right axis.

\section{B. Hole}

While for the electron the barrier has to be taken into account, this is not the case for the hole. Figure 4 shows that the hole is nicely confined within the dot. We therefore only have to consider the contribution of the dot itself. Unfortunately, the complex nature of the valence band makes an intuitive approach to the hole $g$-factor impossible. Where the electron $g$-factor has a clear limit of 2 for infinite quenching, for the hole $g$-factor such a constant limit does not exist. However, with some simple arguments we can at least qualitatively explain our observations.

Due to the symmetry of our dot $(r \gg h)$, the dominating character in the ground state hole wave function is the heavy hole $(\mathrm{HH})$, contributing more than $94 \%$. The orbital momentum of the $\mathrm{HH}$ Bloch state only has a projection along the $z$ axis. A pure $\mathrm{HH}$ state would therefore have $g_{h}^{\|}=0$ and $g_{h}^{\perp} \neq 0$. This explains the large anisotropy between the inplane and out-of-plane hole $g$-factors that we observe in both experiment and calculation: the in-plane $g$-factor is close to zero, while the out-of-plane $g$-factor is more than one order of magnitude larger. As the hole $g$-factor experiences a large shift in the out-of-plane direction, the composition of the ground state must be sensitive to the applied strain. To illustrate this, Fig. 6 therefore shows the calculated contribution of each of the bands to the ground state hole wave function.

The contribution from both the conduction band (CB) and split-off band (SO) are negligible and not sensitive to the applied strain. The $\mathrm{HH}$ contribution increases by $0.25 \%$ which is entirely compensated by an equal decrease in the light hole contribution. Previous research has already shown that the hole $g$-factor is extremely sensitive to this exact composition [8,9]. This therefore qualitatively explains the observed shift. In case of the in-plane hole $g$-factor, strain is affecting something that is close to zero, so in absolute terms the change is small.

\section{CONCLUSIONS}

In this work we have investigated the strain-dependence of the full $g$-tensor for both the electron and hole. We have found a remarkable sensitivity of the out-of-plane hole $g$-factor. This result is consistent in all five investigated QDs and the behavior of all components agrees well with the performed $\boldsymbol{k} \cdot \boldsymbol{p}$ calculations. The electron $g$-tensor was found to show a small increase with applied strain for both components. Anisotropy between the two different components was observed. Both were explained by considering the change in orbital momentum quenching due to the leaking of the wave function into the surrounding barrier. The hole $g$-tensor showed a similar anisotropy in both nominal value and sensitivity, with the large out-of-plane $g$-factor experiencing a large change and the small in-plane $g$-factor experiencing a small change. This was explained by considering the $\mathrm{HH}$-like character of the hole ground state and the change of the LH contribution with applied strain.

As stated in the introduction, tunability of one of the $g$-tensor components around zero would be technologically most relevant. For this purpose, the out-of-plane hole $g$-factor is the most suitable candidate. By size-engineering of the QDs, the nominal value of the $g$-factor, can be brought close to zero, in absence of external strain. Addtionally, a much larger tuning range can be achieved by making use of more advanced devices [38-40], with reported strain values up to $1.5 \%$ [25]. In some of these devices, full anisotropic control over the in-plane strain tensor is possible. This opens up a new way of manipulating the $g$-tensor per component, both in conduction and valence band.

\section{ACKNOWLEDGMENTS}

This work is part of the Graduate Programme of the Netherlands Organisation for Scientific Research (NWO), Project No. 022.005.011. This work has also received financial support from the European Research Council (ERC) under the European Unions Horizon 2020 research and innovation programme, and the Austrian Science Fund (FWF): Grant No. P29603.
[1] P. Michler, Single Semiconductor Quantum Dots (Springer, Berlin/Heidelberg, 2009), pp. 185-225.

[2] D. Loss and D. P. DiVincenzo, Phys. Rev. A 57, 120 (1998).

[3] R. J. Warburton, Nat. Mater. 12, 483 (2013).

[4] R. A. Isaacson, Phys. Rev. 169, 312 (1967).

[5] J. Konopka, Phys. Lett. A 26, 29 (1967).
[6] C. Weisbuch and C. Hermann, Phys. Rev. B 15, 816 (1977).

[7] J. Pingenot, C. E. Pryor, and M. E. Flatteé, Appl. Phys. Lett. 92, 222502 (2008)

[8] J. van Bree, A. Y. Silov, P. M. Koenraad, M. E. Flatté, and C. E. Pryor, Phys. Rev. B 85, 165323 (2012). 
[9] J. van Bree, A. Y. Silov, P. M. Koenraad, and M. E. Flatté, Phys. Rev. Lett. 112, 187201 (2014).

[10] J. van Bree, A. Y. Silov, P. M. Koenraad, and M. E. Flatté, Phys. Rev. B 90, 165306 (2014).

[11] W. Sheng, S. J. Xu, and P. Hawrylak, Phys. Rev. B 77, 241307(R) (2008).

[12] T. Nakaoka, T. Saito, J. Tatebayashi, and Y. Arakawa, Phys. Rev. B 70, 235337 (2004).

[13] J. H. Prechtel, F. Maier, J. Houel, A. V. Kuhlmann, A. Ludwig, A. D. Wieck, D. Loss, and R. J. Warburton, Phys. Rev. B 91, 165304 (2015).

[14] F. Klotz, V. Jovanov, J. Kierig, E. C. Clark, D. Rudolph, D. Heiss, M. Bichler, G. Abstreiter, M. S. Brandt, and J. J. Finley, Appl. Phys. Lett. 96, 053113 (2010).

[15] V. Jovanov, T. Eissfeller, S. Kapfinger, E. C. Clark, F. Klotz, M. Bichler, J. G. Keizer, P. M. Koenraad, G. Abstreiter, and J. J. Finley, Phys. Rev. B 83, 161303(R) (2011).

[16] T. Nakaoka, S. Tarucha, and Y. Arakawa, Phys. Rev. B 76, 041301(R) (2007).

[17] A. J. Bennett, M. A. Pooley, Y. Cao, N. Sköld, I. Farrer, D. A. Ritchie, and A. J. Shields, Nat. Commun. 4, 1522 (2013).

[18] R. S. Deacon, Y. Kanai, S. Takahashi, A. Oiwa, K. Yoshida, K. Shibata, K. Hirakawa, Y. Tokura, and S. Tarucha, Phys. Rev. B 84, 041302(R) (2011).

[19] W. Sheng, Appl. Phys. Lett. 96, 133102 (2010).

[20] R. Trotta, E. Zallo, E. Magerl, O. G. Schmidt, and A. Rastelli, Phys. Rev. B 88, 155312 (2013).

[21] F. Ding, R. Singh, J. D. Plumhof, T. Zander, V. Křápek, Y. H. Chen, M. Benyoucef, V. Zwiller, K. Dörr, G. Bester, A. Rastelli, and O. G. Schmidt, Phys. Rev. Lett. 104, 067405 (2010).

[22] T. Nakaoka, T. Kakitsuka, T. Saito, S. Kako, S. Ishida, M. Nishioka, Y. Yoshikuni, and Y. Arakawa, J. Appl. Phys. 94, 6812 (2003).

[23] S. Seidl, M. Kroner, A. Högele, K. Karrai, R. J. Warburton, A. Badolato, and P. M. Petroff, Appl. Phys. Lett. 88, 203113 (2006).

[24] E. Zallo, R. Trotta, V. Křápek, Y. H. Huo, P. Atkinson, F. Ding, T. Šikola, A. Rastelli, and O. G. Schmidt, Phys. Rev. B 89, 241303(R) (2014).
[25] X. Yuan, F. Weyhausen-Brinkmann, J. Martín-Sánchez, G. Piredda, V. Křápek, Y. Huo, H. Huang, C. Schimpf, O. G. Schmidt, J. Edlinger, G. Bester, R. Trotta, and A. Rastelli, Nat. Commun. 9, 3058 (2018).

[26] H. M. G. A. Tholen, J. S. Wildmann, A. Rastelli, R. Trotta, C. E. Pryor, E. Zallo, O. G. Schmidt, P. M. Koenraad, and A. Y. Silov, Phys. Rev. B 94, 245301 (2016).

[27] J. van Bree, A. Y. Silov, M. L. van Maasakkers, C. E. Pryor, M. E. Flatté, and P. M. Koenraad, Phys. Rev. B 93, 035311 (2016).

[28] M. Bayer, G. Ortner, O. Stern, A. Kuther, A. A. Gorbunov, A. Forchel, P. Hawrylak, S. Fafard, K. Hinzer, T. L. Reinecke, S. N. Walck, J. P. Reithmaier, F. Klopf, and F. Schäfer, Phys. Rev. B 65, 195315 (2002).

[29] L. Wang, A. Rastelli, and O. G. Schmidt, J. Appl. Phys. 100, 064313 (2006).

[30] R. Trotta, P. Atkinson, J. D. Plumhof, E. Zallo, R. O. Rezaev, S. Kumar, S. Baunack, J. R. Schröter, A. Rastelli, and O. G. Schmidt, Adv. Mater. 24, 2668 (2012).

[31] M. Semina and R. Suris, Semiconductors 49, 797 (2015).

[32] I. Vurgaftman, J. R. Meyer, and L. R. Ram-Mohan, J. Appl. Phys. 89, 5815 (2001).

[33] C. Pryor, M.-E. Pistol, and L. Samuelson, Phys. Rev. B 56, 10404 (1997).

[34] C. Pryor, Phys. Rev. B 57, 7190 (1998).

[35] C. E. Pryor and M. E. Flatté, Phys. Rev. Lett. 96, 026804 (2006).

[36] C. E. Pryor and M. E. Flatté, Phys. Rev. Lett. 99, 179901(E) (2007).

[37] V. V. Belykh, D. R. Yakovlev, J. J. Schindler, J. Van Bree, P. M. Koenraad, N. S. Averkiev, M. Bayer, and A. Y. Silov, J. Appl. Phys. 120, 084301 (2016).

[38] R. Trotta, J. Martín-Sánchez, I. Daruka, C. Ortix, and A. Rastelli, Phys. Rev. Lett. 114, 150502 (2015).

[39] R. Trotta, J. Martín-Sánchez, J. S. Wildmann, G. Piredda, M. Reindl, C. Schimpf, E. Zallo, S. Stroj, J. Edlinger, and A. Rastelli, Nat. Commun. 7, 10375 (2016).

[40] J. Martín-Sánchez, R. Trotta, G. Piredda, C. Schimpf, G. Trevisi, L. Seravalli, P. Frigeri, S. Stroj, T. Lettner, M. Reindl, J. S. Wildmann, J. Edlinger, and A. Rastelli, Adv. Opt. Mater. 4, 682 (2016). 